\title{
Orginal Article: \\ The Correlation Between Reach and Grasp Kinematic Measures and Clinical Measures of Manual Dexterity in Patients With Parkinson Disease
}

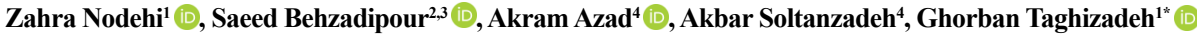

1. Department of Occupational Therapy, School of Rehabilitation Sciences, Iran University of Medical Sciences, Tehran, Iran

2. Department of Mechanical Engineering, Sharif University of Technology, Tehran, Iran.

3. Jawad Movafaghian Research Center in Rehab Technologies, Sharif University of Technology, Tehran, Iran

4. Rehabilitation Research Center, Department of Occupational Therapy, School of Rehabilitation Sciences, Iran University of Medical Sciences, Tehran, Iran

5. Department of Neurology, Shariati Hospital, Tehran University of Medical Sciences, Tehran, Iran.

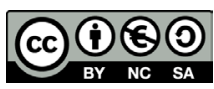

*This work has been published under CC BY-NC-SA 4.0 license.

Article info:

Received: 10 Feb. 2020

Accepted: 15 May 2020

Published: 28 Oct 2020

Funding

The paper was extracted from the MSc. thesis of the first author, Department of Occupational Therapy, School of Rehabilitation Sciences, Iran University of Medical Sciences, Tehran, Iran. This study was supported by the Iran University of Medical Sciences, Tehran, Iran.

Conflict of interest

The authors declared no conflict of interest.

\section{ABSTRACT}

Background and Objectives: To investigate the correlation between reach and grasp kinematic measures and clinical measures of gross and fine manual dexterity in patients with idiopathic Parkinson Disease (PD).

Methods: Sixty patients (44 men, 16 women) with idiopathic PD (Mean \pm SD of age: $59.85 \pm 11.89$ years) were recruited in this non-experimental cross-sectional study using a non-probability sampling method. Their gross and fine manual dexterity were assessed using the Box and Block Test (BBT) and Nine Hole Peg Test (NHPT), respectively. Also, reach and grasp kinematic measures (including normalized movement time, peak velocity, Maximum Grip Aperture [MGA], and percentage of movement time in which MGA occurred [PMGA]) were measured in the more affected hand.

Results: The results showed a significant and moderate correlation between reach kinematic measures (i.e. normalized movement time and peak velocity) and both gross and fine manual dexterity assessed by BBT $(\mathrm{P}=0.000)$ and NHPT $(\mathrm{P}=0.000)$, respectively. However, there was not a significant correlation between grasp kinematic measures (i.e. MGA and PMGA) and gross manual dexterity measured by BBT $(\mathrm{P}>0.05)$ as well as fine manual dexterity assessed by NHPT $(\mathrm{P}>0.05)$.

Conclusion: This study showed no to moderate correlation between reach and grasp kinematic measures and clinical measures of gross and fine manual dexterity in patients with PD, supporting the significance of multi-level evaluation using both clinical and kinematic measures in the evaluation of upper limb function in patients with PD.

Keywords: Manual dexterity, Reach and grasp, Kinematic measures, Parkinson disease

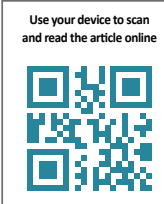

Chtation Nodehi Z, Behzadipour S, Azad A, Soltanzadeh A, Taghizadeh Gh. The Correlation Between Reach and Grasp Kinematic Measures and Clinical Measures of Manual Dexterity in Patients With Parkinson Disease. Function and Disability Journal. 2020; 3:27-34. http://dx.doi.org/10.32598/fdj.3.35.3

doi'http://dx.doi.org/10.32598/fdj.3.35.3

* Corresponding Author:

Ghorban Taghizadeh, PhD.

Address: Rehabilitation Research Center, Department of Occupational Therapy, School of Rehabilitation Sciences, Iran University of Medical Sciences, Tehran, Iran.

Tel: +98 (910) 1462167

E-mail:taghizadeh.gh@iums.ac.ir,ghtaghizade@yahoo.com
What is "already known" in this topic:

Correlation between kinematic parameters and clinical measures of upper limb motor control has been investigated in some health conditions. But it needs to be defined in Parkinson's disease.

$\rightarrow$ What this article adds:

This study shows no to moderate correlation between reach and grasp kinematic parameters and clinical measures. 


\section{Introduction}

$\mathbf{P}$

arkinson Disease (PD) is the second most common age-related neurodegenerative disorder with a prevalence of about $1 \%-2 \%$ in people older than 65 years [1]. Bradykinesia, rigidity, and tremor are cardinal characteristics of $\mathrm{PD}$, affecting the motor skills of the patient, especially those needing coordination such as manual dexterity [2]. Patients with PD suffer from different sensory deficits in addition to motor impairments (e.g. impairments of proprioception, perception of object weight) [3], vibration, discriminative sense [4], etc.), which may also be involved in manual dexterity impairments.

Manual dexterity is defined as goal-directed activities, involving reaching, grasping, and coordinated and controlled movements with hands and fingers that is an important part of many Activities of Daily Living (ADLs) [5]. Patients with PD commonly experience impairments of manual dexterity, leading to decreased independence in performing ADLs. Thus, precise and efficient assessment of manual dexterity in patients with PD can not only enable a comprehensive evaluation of improvement following rehabilitation/medication intervention, but also help clinicians to individually adapt medication doses and select appropriate rehabilitation interventions [6]. Different functional, clinical, and laboratory assessment methods have been proposed to evaluate manual dexterity. Traditionally, the manual dexterity of patients with PD has been assessed by functional/clinical measures such as Box and Block Test (BBT), Nine Hole Peg Test (NHPT), and so on that are inexpensive and simple assessment tools designed for evaluating fine motor functions. Although these tests include hand-object interactions and give the examiner a global outcome measurement of upper limb function, they have some limitations including subjectivity and poor sensitivity to mild impairments [7]. Besides, detailed important information about the associated kinematic variables (e.g. acceleration and velocity) and different movement phases cannot be provided by clinical measures.

Conversely, laboratory tools such as motion analysis system can be used for quantitative kinematic analysis, which provides useful and important information about both spatial and temporal aspects of upper limb movements [8]. Hence, therapists/researchers would benefit from the objective evaluation of upper limb function based on kinematic parameters using a motion analysis system. Although it seems that laboratory measures such as kinematic parameters determined by motion analysis system are more representative of motor control strate- gies, only a few studies have evaluated the relationship between upper limb kinematic parameters and clinical data. Also these studies were limited to the subjects with stroke [9-11]. Only recently, Garza Rodriguez et al. reported a significant correlation between kinematic measures of supination and pronation and clinical assessment of supination/pronation using item 3.6 of the Unified Parkinson's Disease Rating Scale (UPDRS) [12].

However, understanding the correlations between kinematic parameters and clinical measures would allow the recognition of kinematic parameters that best indicate sensorimotor impairment, resulting in better clinical interpretation of kinematic parameters. Moreover, investigating the correlations between kinematic parameters and clinical measures in patients with PD is important to assess the suitability and efficacy of a clinical measure to evaluate upper limb motor control in these patients. Also, in addition to providing abundant areas for future experimental research, the correlational studies enable predicting the scores of one measure based on another measure [13]. Thus, this study aimed to determine the extent to which the reach and grasp kinematic measures obtained using a motion analysis system are associated with gross and fine manual dexterity as assessed by clinical tests in patients with PD.

\section{Materials and Methods}

\section{Study participants}

Sixty patients with idiopathic PD (44 men, 16 women) with the Mean \pm SD age of $63.90 \pm 9.38$ years participated in this non-experimental cross-sectional study. They were recruited by simple non-probability method from movement disorders clinics in Tehran based on the following inclusion criteria: having PD based on the neurologist diagnosis with an acceptable level of cognitive function (i.e. the score $>23$ on the Mini-Mental Status Examination [MMSE]) [14], and lacking other neurological diseases such as multiple sclerosis and stroke, orthopedic problems such as tendon injuries or fracture in the upper extremity. Subjects were excluded in the case of lack of cooperation in the execution of the tests or the inability to do the tests. The severity of motor symptoms was evaluated by the motor subsection of the UPDRS (i.e. UPDRS-III). The more affected side was determined based on the score on the 20-26 items of UPDRS-III. Before participation in the study, a written consent form was signed by all patients which was approved by the Ethics Committee of Iran University of Medical Sciences. 


\section{Study procedure}

The general and clinical characteristics of the participants including sex, age, dominant hand, more affected hand, and time since diagnosis of PD were recorded using a demographic questionnaire. The following assessments were conducted in random order by a trained examiner in a single day and during the on-medication phase (i.e. 1-2 hours after taking dopaminergic medication) [15]. Sufficient rest periods were given to the patients between evaluations to prevent fatigue.

Gross and fine manual dexterity were assessed by BBT and NHPT, respectively. BBT is a well-accepted measure of gross manual dexterity, in which the subject is instructed to move wooden blocks from one compartment to the other compartment of the box by one hand during one minute. The test is conducted for both hands and the score of each hand is equal to the number of blocks moved in one minute by that hand [16]. This test has good validity and high reliability [17]. The BBT score of the more affected hand was used in this study.

NHPT is a test for evaluating fine manual dexterity and contains nine pegs that must be inserted and removed by one hand as quickly as possible into nine holes on the test board. NHPT is executed two times by each hand and the mean score of the two trials is recorded as the NHPT score for the respected hand [18]. A high test-retest reliability has been reported for the NHPT in patients with PD [19]. The NHPT score of the more affected hand was used in the current study.

The kinematic assessment was done using a 6-camera motion analysis system (VICON 2.5, VICON, UK) at $120 \mathrm{~Hz}$ and included a task of reaching, grasping, and lifting a jar. This task was specifically chosen due to its fundamental role in performing different ADLs [20]. During the kinematic evaluation, the subject sat on a chair in front of a table, where the target (i.e. the jar) was placed along the body midline of the subject. The reaching distance to the target was equal to the maximum reachable distance for each participant and the task was performed by the more affected hand. One practice trial and two main trials were done. Reflective markers were attached to the styloid process of the ulna and the nail of thumb and index fingers, as well as the jar. The obtained kinematic data were analyzed using a custom code written in MATLAB R2013 and then reach (i.e. normalized movement time and peak velocity) and grasp (i.e. Maximum Grip Aperture [MGA] and the percentage of movement time in which MGA occurs [PMGA]) kinematic parameters were calculated. To adjust varia- tions in the reaching distance, the movement time which is considered as a measure of movement efficiency was normalized to the reaching distance for each participant [21]. The peak velocity indicates the force at the movement initiation and skillful/pre-programmed movement initiation is usually associated with greater peak velocity. MGA is an index of spatial control of grasp and is defined as the maximum distance between the thumb and index finger tips. PMGA, an index of the temporal control of grasp, indicates the correction of the hand opening signal while the hand approaches the target $[8,22]$.

\section{Statistical analysis}

The normal distribution of the data was investigated by the Shapiro-Wilks test $(\mathrm{P}>0.05)$. The Pearson and Spearman correlation coefficients were used to investigate the correlation between different kinematic measures and gross and fine manual dexterity. The strength of the correlation was determined as following: coefficient values of 0.90-1.00, 0.70-0.89, 0.50-0.69, 0.26-0.49, and 0.000.25 indicate very high, high, moderate, low, and little (if any) correlation, respectively [13]

\section{Results}

\section{Participants' characteristics}

Table 1 presents the demographic and clinical characteristics of the participants. The Mean \pm SD of age, time since PD diagnosis, and UPDRS-III score of the participants were $63.90(9.38)$ years, $7.13(5.01)$ years, and 18.80 (10.45), respectively. All participants were righthanded. The number of patients with right or left more affected hand was equal. The Mean \pm SD of reach and grasp kinematic measures, including normalized movement time $(\mathrm{s})$, peak velocity $(\mathrm{cm} / \mathrm{s})$, MGA $(\mathrm{cm})$, and PMGA (\%) were 0.05 (0.3), 82.63 (31.43), 12.76 (2.00), 82.82 (10.43), respectively. The Mean \pm SD of BBT and NHPT scores was 51.14 (11.52) and 28.98 (12.27), respectively.

The Correlation between reach and grasp kinematic measures and gross manual dexterity in patients with PD

The results of this study revealed a significant and moderate correlation between reach kinematic measures (i.e. normalized movement time and peak velocity) and gross manual dexterity assessed by BBT $(\mathrm{P}=0.000)$. However, there was not a significant correlation between grasp kinematic measures (i.e. MGA and PMGA) and gross manual dexterity measured by BBT $(\mathrm{P}>0.05)$ (Table 2$)$. 
The Correlation between reach and grasp kinematic measures and fine manual dexterity in patients with PD

There was a significant and moderate correlation between reach kinematic measures (i.e. normalized movement time and peak velocity) and fine manual dexterity evaluated by NHPT $(\mathrm{P}=0.000)$. However, grasp kinematic measures (i.e. MGA and PMGA) were not significantly correlated with fine manual dexterity measured by NHPT $(\mathrm{P}>0.05)$ (Table 3$)$.

\section{Discussion}

Three cardinal characteristics of PD, including bradykinesia, rigidity, and tremor results in impairments of manu- al dexterity that are commonly exhibited as timing, speed, and force modulation, disturbing quality of hand functions, and ADLs progressively throughout the disease. These impairments may result in using compensatory strategies or avoiding specific tasks entirely due to fear of injury or embarrassment [23]. Thus, evaluation of manual dexterity is of particular importance in patients with PD. Traditionally, clinical measures such as BBT and NHPT are used to assess gross and fine manual dexterity, respectively. Kinematic evaluations may identify changes in manual dexterity that are not provided by only considering the time of completing the NHPT or the number of blocks transferred in BBT. Besides, they give objective parameters that may be more sensitive and not susceptible to ceiling effects [24]. The significant correlation between upper limb kinematic measures and clini-

Table 1. Demographic and clinical characteristics of the participants $(n=60)$

\begin{tabular}{cc}
\hline Variables & No. (\%)/ Mean \pm SD \\
\hline Gender (male/female) & $44.16(73.3 / 26.7)$ \\
\hline Dominant hand (right/left) & $60.0(100 / 0)$ \\
\hline More affected hand (right/left) & $30.30(50 / 50)$ \\
Age (y) & $63.90 \pm 9.38$ \\
\hline Time since PD diagnosis (y) & $7.13 \pm 5.01$ \\
UPDRS-III (score) & $18.80 \pm 10.45$ \\
\hline
\end{tabular}

UPDRS-III: The motor subsection of Unified Parkinson's Disease Rating Scale

Table 2. The correlation between reach and grasp kinematic measures and gross manual dexterity in patients with PD

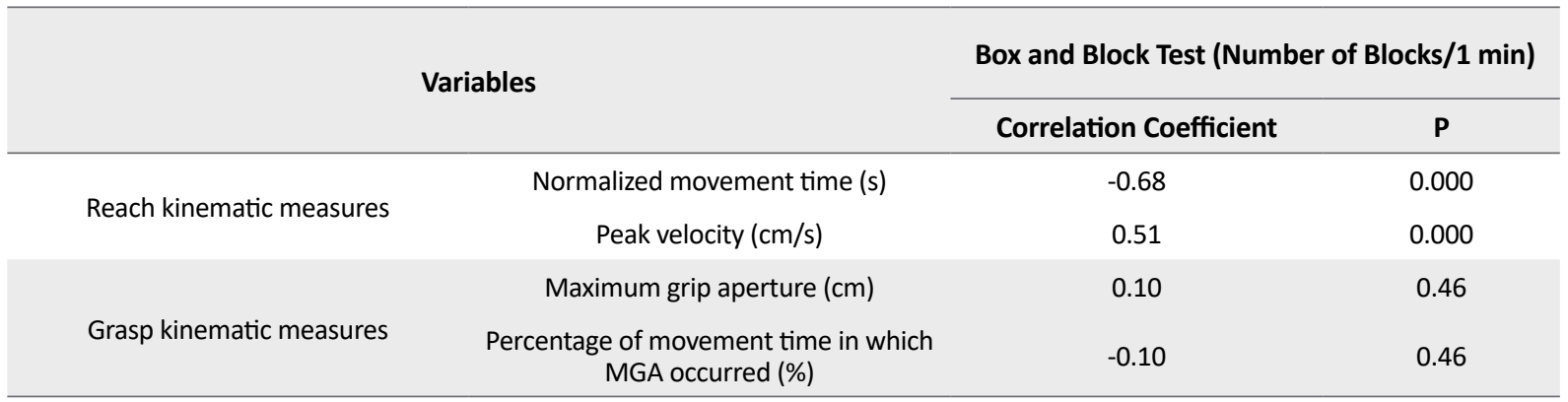

Table 3. The correlation between reach and grasp kinematic measures and fine manual dexterity in patients with PD

\begin{tabular}{|c|c|c|c|}
\hline & \multirow{2}{*}{ Variables } & \multicolumn{2}{|c|}{ Nine Hole Peg Test (Second) } \\
\hline & & Correlation Coefficient & $\mathbf{P}$ \\
\hline \multirow{2}{*}{$\begin{array}{l}\text { Reach kinematic } \\
\text { measures }\end{array}$} & Normalized movement time (s) & 0.65 & 0.000 \\
\hline & Peak velocity $(\mathrm{cm} / \mathrm{s})$ & -0.50 & 0.000 \\
\hline \multirow{2}{*}{$\begin{array}{l}\text { Grasp kinematic } \\
\text { measures }\end{array}$} & Maximum grip aperture $(\mathrm{cm})$ & -0.23 & 0.08 \\
\hline & $\begin{array}{l}\text { Percentage of movement time in which } \\
\text { MGA occurred (\%) }\end{array}$ & 0.20 & 0.13 \\
\hline
\end{tabular}


cal measures of manual dexterity (i.e. BBT and NHPT) has been previously reported in other neurological disorders than PD $[9,11,24]$. This is the first study, to the best of authors' knowledge, which investigated the correlation between reach and grasp kinematic measures and manual dexterity in patients with PD.

The results of this study showed that reach the kinematic measure of normalized movement time was moderately and negatively correlated with the BBT score while it was moderately and positively correlated with the NHPT score. Moreover, the results of the current study revealed that the reach kinematic measure of peak velocity was moderately and positively correlated with the BBT score, whereas it was moderately and negatively correlated with the NHPT score. These findings indicate that the shorter reaching time and the higher reaching peak velocity is associated with greater gross and fine manual dexterity measured by BBT and NHPT, respectively. One possible explanation for these findings may be the time dependency of both BBT and NHPT which mainly indicates the velocity of motor function. Therefore, the high correlation may be found using tests that measure other aspects of motor function. In line with the results of the current study, Adams et al. also found a significant correlation between upper limb kinematic measures (i.e. normalized speed) and BBT score in stroke survivors [9]. Gomes et al. (2020) also reported a significant correlation between movement time in a reach and grasp task and gross manual dexterity assessed by the BBT in stroke survivors [11]. A significant correlation between kinematic measure (i.e. total movement time) and NHPT score has also been found by Johansson and Häger in stroke survivors [24].

Conversely, there was not a significant correlation between grasp kinematic measures (i.e. MGA and PMAG) and clinical measures of gross and fine manual dexterity. As mentioned previously, kinematic evaluations are assumed to give objective and sensitive parameters without ceiling effect [24, 25], while clinical measures usually have ordinal nature and ceiling effects [25]. Thus, it is expected that kinematic parameters yield complementary information to clinical measures that may result in less than a high correlation between reach and grasp kinematic parameters and clinical measures [25]. Nevertheless, sensory and motor deficits occurred in PD may affect other aspects of upper limb motor function than speed [26]. Thus, clinical measures that evaluate different aspects of upper limb motor function, including grasp quality may show a significant correlation with grasp kinematic measures.

Finally, some limitations of this study should be considered. First, the inclusion criteria only allowed participation of patients without cognitive impairment. Thus, the findings of the current study may not be generalizable to patients with more advanced PD who have cognitive impairments. Second, due to the ethical issues, participants were only evaluated in the on-medication phase. However, investigating the correlation between reach and grasp kinematic measures and clinical measures of manual dexterity may provide a more comprehensive understanding of the correlation between kinematic measures and manual dexterity in these patients that are suggested to be addressed in future studies.

\section{Conclusion}

The current study results indicated that reach kinematic measures of normalized movement time and peak velocity, but not grasp kinematic measures, are significantly and moderately correlated with both gross and fine manual dexterity in patients with PD. Therefore, reach and grasp kinematic measures and clinical measures may evaluate somewhat different aspects of manual dexterity. Hence, it can be suggested that using both laboratory and clinical measures, whenever possible, would enhance the comprehensiveness of manual dexterity assessment in patients with PD.

The results of this study support the need for the multilevel assessment using both clinical measures and kinematic parameters in the evaluation of upper limb function in patients with PD.

\section{Ethical Considerations}

\section{Compliance with ethical guidelines}

This study was approved by the Ethics Committee of the Iran University of Medical Sciences.

\section{Funding}

The paper was extracted from the MSc. thesis of the first author, Department of Occupational Therapy, School of Rehabilitation Sciences, Iran University of Medical Sciences, Tehran, Iran. This study was supported by the Iran University of Medical Sciences, Tehran, Iran.

\section{Authors' contributions}

Conceptualization, supervision: Ghorban Taghizadeh; Methodology: Ghorban Taghizadeh, Saeed Behzadipour, Akram Azad, Akbar Soltanzadeh; Investigation: Zahra Nodehi; Writing - original draft; writing - review \& editing: All authors; Funding acquisition, resources: Ghorban Taghizadeh. 


\section{Conflict of interest}

The authors declared no conflict of interest.

\section{References}

[1] Adams RJ, Ellington, AL, Armstead K, Sheffield K, Patrie JT, Diamond PT. Upper extremity function assessment using a glove orthosis and virtual reality system. OTJR (Thorofare N J). 2019; 39(2):81-9. [DOI:10.1177/1539449219829862] [PMID] [PMCID]

[2] Carr JH, Shepherd RB. Stroke rehabilitation: Guidelines for exercise and training to optimize motor skill. Massachusetts: Butterworth-Heinemann Medical; 2003. https://books.google.com/ books?id=AccPvgAACAAJ\&dq

[3] de los Reyes-Guzmán A, Dimbwadyo-Terrer I, Trincado-Alonso F, Monasterio-Huelin F, Torricelli D, et al. Quantitative assessment based on kinematic measures of functional impairments during upper extremity movements: A review. Clin Biomech (Bristol, Avon). 2014; 29(7):719-27. [DOI:10.1016/j.clinbiomech.2014.06.013] [PMID]

[4] Desrosiers J, Bravo G, Hébert R, Dutil E, Mercier L. (1994). Validation of the Box and Block Test as a measure of dexterity of elderly people: reliability, validity, and norms studies. Arch Phys Med Rehabil. 75(7):751-5. [DOI:10.1016/0003-9993(94)90130-9] [PMID]

[5] Carter R, Lubinsky J. Rehabilitation research: Principles and applications. Elsevier Health Sciences; 2015. https://books.google.com/book $\mathrm{s} ? \mathrm{id}=7 \mathrm{osNCgAAQBAJ} \&$ printsec $=$ frontcover\&dq

[6] Earhart GM, Cavanaugh JT, Ellis T, Ford MP, Foreman KB, Dibble L. The 9-hole PEG test of upper extremity function: Average values, test-retest reliability, and factors contributing to performance in people with Parkinson disease. J Neurol Phys Ther. 2011; 35(4):157-63. [DOI:10.1097/NPT.0b013e318235da08] [PMID]

[7] Eder CF, Popović MB, Popović DB, Stefanović A, Schwirtlich L, Jović S. The drawing test: Assessment of coordination abilities and correlation with clinical measurement of spasticity. Arch Phys Med Rehabil. 2005; 86(2):289-95. [DOI:10.1016/j.apmr.2004.04.045] [PMID]

[8] Foki T, Pirker W, Geißler A, Haubenberger D, Hilbert M, Hoellinger I, et al. Finger dexterity deficits in Parkinson's disease and somatosensory cortical dysfunction. Parkinsonism Relat Disord. 2015; 21(3):259-65. [DOI:10.1016/j.parkreldis.2014.12.025] [PMID]

[9] Garza-Rodríguez A, Sánchez-Fernández LP, Sánchez-Pérez LA, Ornelas-Vences C, Ehrenberg-Inzunza M. Pronation and supination analysis based on biomechanical signals from Parkinson's disease patients. Artif Intell Med. 2018; 84:7-22. [DOI:10.1016/j.artmed.2017.10.001] [PMID]

[10] Godefroy O, Fickl A, Roussel M, Auribault C, Bugnicourt JM, Lamy $\mathrm{C}$, et al. Is the montreal cognitive assessment superior to the mini-mental state examination to detect poststroke cognitive impairment? A study with neuropsychological evaluation. Stroke. 2011; 42(6):1712-6. [DOI:10.1161/STROKEAHA.110.606277] [PMID]

[11] Gomes CLA, Cacho RO, Nobrega VTB, de A Confessor EM, de Farias EEM, Neto JLF, et al. Low-cost equipment for the evaluation of reach and grasp in post-stroke individuals: A pilot study.
Biomed Eng Online. 2020; 19(1):14. [DOI:10.1186/s12938-0200758-7] [PMID] [PMCID]

[12] Kandel ER, Schwartz JH, Jessell T, Siegelbaum S, Hudspeth AJ. Principles of neural science. McGraw-Hill Education; 2012. https://books.google.com/books?id=s64z-LdAIsEC\&q

[13] Johansson GM, Häger CK. A modified standardized nine hole peg test for valid and reliable kinematic assessment of dexterity post-stroke. J Neuroeng Rehabil. 2019; 16(1):8. [DOI:10.1186/ s12984-019-0479-y] [PMID] [PMCID]

[14] Kalkan AC, Kahraman T, Ugut BO, Colakoglu BD, Genc A. A comparison of the relationship between manual dexterity and postural control in young and older individuals with Parkinson's disease. J Clin Neurosci. 2020; 75:89-93. [DOI:10.1016/j. jocn.2020.03.018] [PMID]

[15] Levin MF. Interjoint coordination during pointing movements is disrupted in spastic hemiparesis. Brain. 1996; 119(Pt 1):28193. [DOI:10.1093/brain/119.1.281] [PMID]

[16] Lin KC, Wu CY, Wei TH, Lee CY, Liu JS. Effects of modified constraint-induced movement therapy on reach-to-grasp movements and functional performance after chronic stroke: A randomized controlled study. Clin Rehabil. 2007; 21(12):1075-86. [DOI:10.1177/0269215507079843] [PMID]

[17] Liu CC, Li CY, Lee PC, Sun Y. Variations in incidence and prevalence of Parkinson's Disease in Taiwan: A populationbased nationwide study. Parkinsons Dis. 2016; 2016:8756359. [DOI:10.1155/2016/8756359] [PMID] [PMCID]

[18] Liubicich ME, Magistro D, Candela F, Rabaglietti E, Ciairano S. Physical activity, fine manual dexterity and a coach's selfefficacy in a physical activity program for older persons living in residential care facilities. Psychology. 2012; 3(5):384-92. [DOI:10.4236/psych.2012.35054]

[19] Maschke M, Tuite PJ, Krawczewski K, Pickett K, Konczak J. Perception of heaviness in Parkinson's Disease. Mov Disord. 2OO6; 21(7), 1013-8. [DOI:10.1002/mds.20876] [PMID]

[20] Morris S, Morris ME, Iansek R. Reliability of measurements obtained with the Timed "Up \& Go" test in people with Parkinson disease. Phys Ther. 2001; 81(2):810-8. [DOI:10.1093/ $\mathrm{ptj} / 81.2 .810]$ [PMID]

[21] Nolano M, Provitera V, Estraneo A, Selim MM, Caporaso G, Stancanelli A, et al. Sensory deficit in Parkinson's disease: Evidence of a cutaneous denervation. Brain. 2008; 131(7):1903-11. [DOI:10.1093/brain/awn102] [PMID]

[22] Piro NE, Piro LK, Kassubek J, Blechschmidt-Trapp RA. Analysis and visualization of 3D motion data for UPDRS rating of patients with Parkinson's disease. Sensors (Basel). 2016; 16(6):930. [DOI:10.3390/s16060930] [PMID] [PMCID]

[23] Quinn L, Busse M, Dal Bello-Haas V. Management of upper extremity dysfunction in people with Parkinson disease and Huntington disease: facilitating outcomes across the disease lifespan. J Hand Ther. 2013; 26(2):148-54. [DOI:10.1016/j.jht.2012.11.001] [PMID]

[24] Schwarz A, Kanzler CM, Lambercy O, Luft AR, Veerbeek JM Systematic review on kinematic assessments of upper limb movements after stroke. Stroke. 2019; 50(3):718-27. [DOI:10.1161/ STROKEAHA.118.023531] [PMID]

[25] Slota GP, Enders LR, Seo NJ. Improvement of hand function using different surfaces and identification of difficult move- 
ment post stroke in the Box and Block Test. Appl Ergon. 2014; 45(4):833-8. [DOI:10.1016/j.apergo.2013.10.014] [PMID]

[26] Wu CY, Chen CL, Tang SF, Lin KC, Huang YY. Kinematic and clinical analyses of upper-extremity movements after constraintinduced movement therapy in patients with stroke: A randomized controlled trial. Arch Phys Med Rehabil. 2007; 88(8):964-70. [DOI:10.1016/j.apmr.2007.05.012] [PMID] 


\title{
ارتباط بين معيارهاى كينماتيك دراز كردن دست و گرفتن و معيار هاى بالينى مهارت دستى درشت

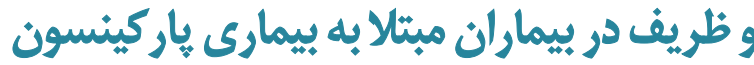

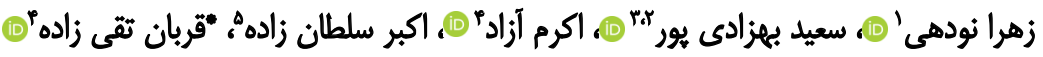 \\ ا. ا.كروه كاردرمانى، دانشكده علوم توانبخشى، دانشكاه علوم يزشكى ايران، تهران، ايران.

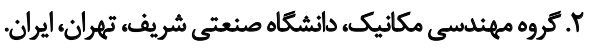

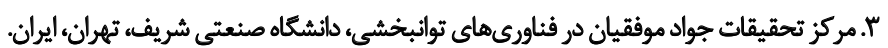

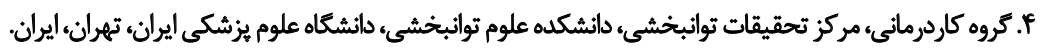

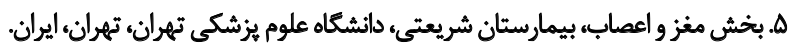

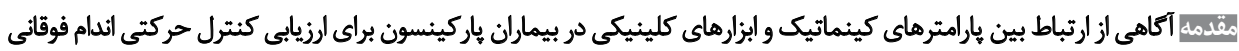

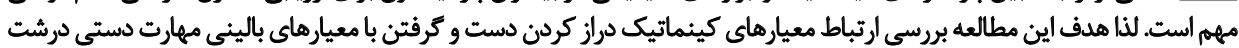

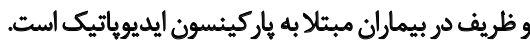

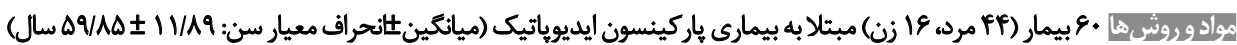

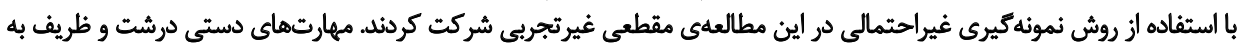

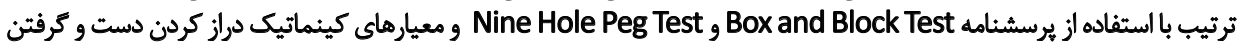

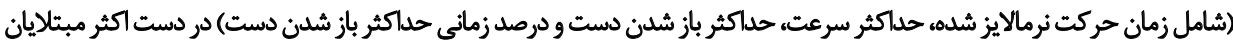
ارزيابي شد.
\end{abstract}

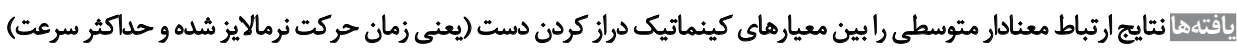

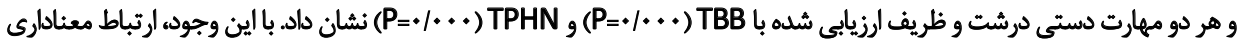

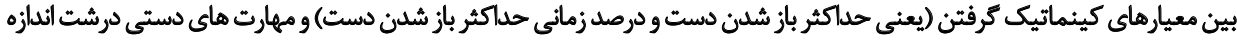

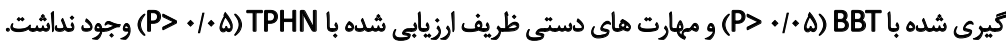

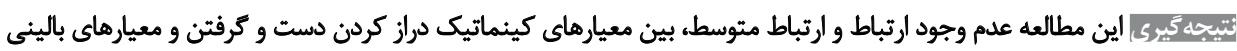

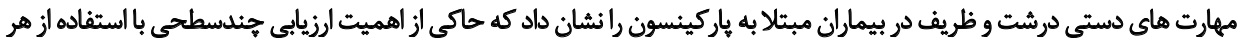

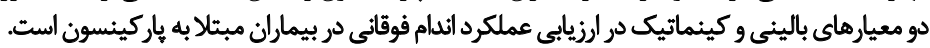
matic Measures and Clinical Measures of Manual Dexterity in Patients With Parkinson Disease. Function and Disability Journal. 2020; 3:27-34. http://dx.doi.org/10.32598/fdj.3.5

doil'http://dx.doi.org/10.32598/fdj.3.5 